\title{
Thermal activation energy for the passivation of the $n$-type crystalline silicon surface by hydrogenated amorphous silicon
}

\author{
Jonathon Mitchell, ${ }^{\text {a) }}$ Daniel Macdonald, and Andres Cuevas \\ School of Engineering, The Australian National University, Canberra, Australian Capital Territory 0200, \\ Australia
}

(Received 28 January 2009; accepted 22 March 2009; published online 20 April 2009)

\begin{abstract}
Excellent surface passivation of crystalline silicon wafers is known to occur following post-deposition thermal annealing of intrinsic $a-\mathrm{Si}: \mathrm{H}$ thin-film layers deposited by plasma-enhanced chemical vapor deposition. In this work, layer thicknesses ranging from 5 to 50 $\mathrm{nm}$ were used to indirectly study the surface passivation mechanism by sequentially measuring the effective carrier lifetime as a function of annealing time and temperature. From this, an activation energy of $0.7 \pm 0.1 \mathrm{eV}$ was calculated, suggesting that surface passivation is reaction-limited and not determined by a bulk hydrogen diffusion process. We conclude that the primary surface reaction stems from surface rearrangement of hydrogen already near the interface. (C) 2009 American Institute of Physics. [DOI: 10.1063/1.3120765]
\end{abstract}

Hydrogenated amorphous silicon $(a-\mathrm{Si}: \mathrm{H})$ has recently been shown to provide an excellent alternative to hightemperature junction formation on crystalline silicon $(c-\mathrm{Si})$ for solar cells. ${ }^{1}$ However, the performance of this material in heterojunctions remains dependent upon the recombination at the $a-\mathrm{Si}: \mathrm{H} / c-\mathrm{Si}$ interface. It is known that deep trapping sites (i.e., dangling bonds) at the $c$-Si surface act as recombination centers for photogenerated carriers from the base wafer. Hydrogen has been shown to passivate these bonds effectively using hydrogenated material including $a-\mathrm{Si}: \mathrm{H}^{2}$ and $a-\mathrm{SiN}: \mathrm{H}^{3}$ The interaction of hydrogen from the $a-\mathrm{Si}: \mathrm{H}$ layer with the $c$-Si surface is of particular importance for improving surface passivation and overall solar cell performance. In the past, hydrogen has been assumed to diffuse from within the thin-film bulk layer toward the $c$-Si surface, whereby passivation occurs. ${ }^{2,4-6}$ However, bonded hydrogen requires a minimum $2.49 \mathrm{eV}^{7}$ for release, leading to the conclusion that hydrogen must either already be in a free or quasi-free state. The high dangling bond density makes the first possibility unlikely, leading Pantelides ${ }^{8}$ to conclude that loosely bonded hydrogen was capable of diffusion by "bond interconversion" with an energy of $1.5 \mathrm{eV}$. This represented the most likely bulk diffusion mechanism for hydrogen. However, low temperature thermal annealing and a relatively high defect density $\left[10^{15}-10^{17} \mathrm{~cm}^{-38}\right]$ would significantly inhibit a bulk diffusion of hydrogen toward the interface for surface passivation.

In this letter, we report our work on the study of the surface passivation mechanism by effective carrier lifetime $\left(\tau_{\mathrm{eff}}\right)$ measurements of the surface passivation quality by contactless photoconductance decay (PCD) ${ }^{9}$ following incremental post-deposition thermal annealing below $300{ }^{\circ} \mathrm{C}$. Here, $1.4 \Omega \mathrm{cm} c-\operatorname{Si}(n)\langle 100\rangle \mathrm{FZ}$ wafers were $\mathrm{HNO}_{3} / \mathrm{HF}$ etched to an average thickness of $220 \mu \mathrm{m}$. Wafers were rinsed and dried following a standard RCA cleaning/HF-dip step, immediately prior to the deposition of intrinsic $a-\mathrm{Si}: \mathrm{H}$ layers $[a-\mathrm{Si}: \mathrm{H}(i)]$ at $5 \mathrm{~W}$, using a $13.56 \mathrm{MHz}$ parallel-plate plasma-enhanced chemical vapor deposition (PECVD) reac-

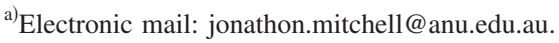

tor. In this system, $c$-Si wafers are suspended from the top heating electrode such that the $a-\mathrm{Si}: \mathrm{H}$ layer deposition occurs on the underside surface. Unwanted plasma components and particulates are removed from the system away from the deposition area. All depositions were performed at $225^{\circ} \mathrm{C}$. We found that postdeposition thermal annealing was essential for improving the poor as-deposited surface passivation quality. Relatively thin (from 5 to $100 \mathrm{~nm}$ ) layers were deposited and $\tau_{\text {eff }}$ was measured at room temperature and at an excess carrier density of $\Delta n=1 \times 10^{15} \mathrm{~cm}^{-3}$ following sequential $10 \mathrm{~min}$ thermal annealing steps.

In Fig. 1, the quality of the surface passivation provided by $a-\mathrm{Si}: \mathrm{H}(i)$ can be seen to improve significantly following post-deposition thermal annealing from an otherwise poorly passivated surface. This improved surface passivation remains stable following an hour long illumination with white light at an intensity of $100 \mathrm{~mW} / \mathrm{cm}^{2}$. Also, it can be seen that the annealing temperature determines the rate of surface passivation reaction, implying a thermally activated reaction for the surface passivation mechanism. The lower, $185^{\circ} \mathrm{C}$ anneal requires a significantly longer anneal time compared to higher temperature anneals near or above the deposition temperature.

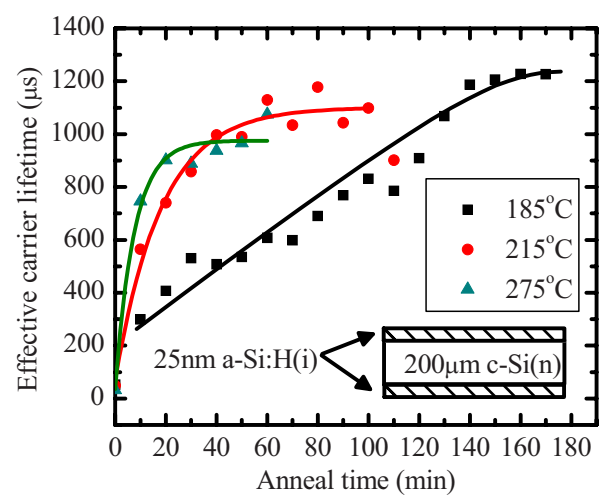

FIG. 1. (Color online) Measured annealing time dependence of the $\tau_{\text {eff }}$ for $25 \mathrm{~nm}$ thick $a$-Si: $\mathrm{H}(i)$ films, for three different annealing temperatures. The results indicate that the reaction rate for surface passivation increases with higher temperature. Fitted lines are shown. 


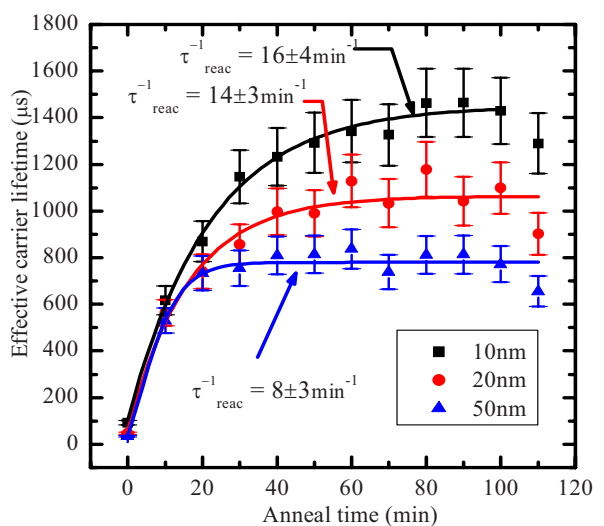

FIG. 2. (Color online) Improvements in $\tau_{\text {eff }}$ from post-deposition thermal annealing for three different thicknesses of as-deposited $a-\mathrm{Si}: \mathrm{H}(i)$. Sigmoidal fits and reaction rate (in minutes) for each thickness are shown.

Anneal temperatures near or higher than $225^{\circ} \mathrm{C}$ were observed to accelerate the passivation reaction, and the "saturation" point occurs earlier. The reaction rate $\left(1 / \tau_{\text {reac }}\right)$ of this improved surface passivation was determined by fitting Eq. (1), for the measured $\tau_{\text {eff }}$ at room temperature following each $10 \mathrm{~min}$ anneal. Here, $\tau_{\text {reac }}$ is the reaction time constant, $\tau_{B}$ is the bulk lifetime, $A$ is a pre-exponential factor and $t$ is the anneal time,

$$
\tau_{\text {eff }}=\left[1 / \tau_{B}+A \exp \left(-t / \tau_{\text {reac }}\right)\right]^{-1} .
$$

The process is illustrated in Fig. 2, for three $a-\mathrm{Si}: \mathrm{H}(i)$ layers thicknesses annealed at $225^{\circ} \mathrm{C}$.

The similar reaction rates for different $a-\mathrm{Si}: \mathrm{H}(i)$ layer thicknesses would suggest that surface passivation was independent of a bulk diffusion mechanism. The reaction rates $\left(1 / \tau_{\text {reac }}\right)$ were then plotted against inverse temperature. In Fig. 3, the results for the $25 \mathrm{~nm}$ thick $a$-Si:H layer are shown. The error bars represent the maximum and minimum fitted solution of $1 / \tau_{\text {reac }}$ to the measured $\tau_{\text {eff }}$. The activation energy $\left(E_{A}\right)$ of the surface passivation mechanism can be determined from the slope of the fit according to Eq. (2), where $k_{B}$ is the Boltzmann constant and $T$ is the temperature,

$$
1 / \tau_{\text {reac }}=A \exp \left[-E_{A} / k_{B} T\right]
$$

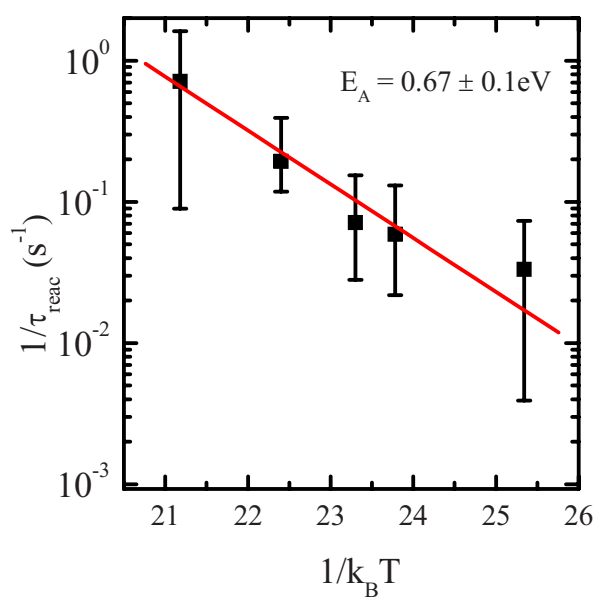

FIG. 3. (Color online) Arrhenius plot of reaction rate showing the fit for determining the activation energy of this process. A $25 \mathrm{~nm} a-\mathrm{Si}: \mathrm{H}(i)$ layer deposited onto a $200 \mu \mathrm{m} c$ - $\operatorname{Si}(n)$ wafer is shown. Fitted line according to Eq. (2).

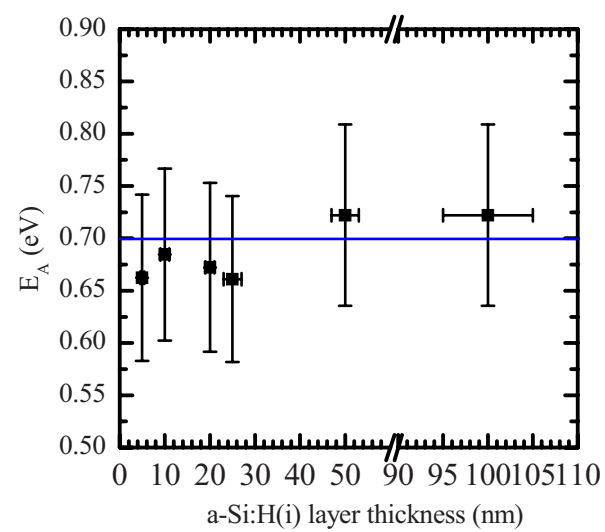

FIG. 4. (Color online) Calculated thermal surface passivation activation energies for different $a$-Si:H( $i)$ layer thicknesses. Dotted line indicates the average value for $E_{A}$ across all thicknesses.

The process was repeated for $a-\mathrm{Si}: \mathrm{H}(i)$ layer thicknesses between 5 and $100 \mathrm{~nm}$. The activation energy does not have a strong dependence on thickness, as illustrated by Fig. 4 .

An averaged value of $0.7 \pm 0.1 \mathrm{eV}$ for the activation energy of surface passivation was calculated for the $1.4 \Omega \mathrm{cm}$ $c-\mathrm{Si}(n)\langle 100\rangle F Z$ surface across the $a$-Si:H $(i)$ layer thicknesses tested in this work. De Wolf and Kondo ${ }^{10}$ have reported a similar value for $E_{A}$ of $0.6 \mathrm{eV}$ for $a-\mathrm{Si}: \mathrm{H}$ films deposited at lower temperatures than those used here. Biegelson et al. ${ }^{11}$ has previously reported a similar $E_{A}$ from electron-spin resonance measurements with thermal annealing up to $250{ }^{\circ} \mathrm{C}$ with a parallel reduction in $\mathrm{Si}$ dangling bond density at the $c$-Si surface.

The value for $E_{A}$ determined in this work differs from those reported for bulk-related hydrogen diffusion processes in $a-\mathrm{Si}: \mathrm{H}$ of approximately $1.5 \mathrm{eV}^{8}$ and the $2.49 \mathrm{eV}^{7} \mathrm{re}-$ quired for spontaneous hydrogen release. The lower $E_{A}$ determined in this work indicates that surface passivation is primarily dependent upon surfaces states, rather than bulk diffusion processes. This would indicate that the surface passivation mechanism was not a bulk related mechanism. One possible explanation is that this lower activation energy reflects the chemical rearrangement of hydrogen already present at the interface prior to deposition (HF-dip step) or else from hydrogen deposited during the initial stages of deposition.

The complexity in studying the surface itself in combination with a limited analysis being performed at higher temperatures $\left(\geq 400{ }^{\circ} \mathrm{C}\right.$ ) has led others to conjecture that hydrogen diffuses from the thin-film bulk toward the surface. ${ }^{2,12-15}$ While hydrogen diffusion can of course occur at higher temperature, our results indicate that an alternative surface reaction-based mechanism, involving hydrogen already very close to the interface, is in fact responsible for the passivation of the $c$-Si surface. This also solves the problem of hydrogen diffusing from the thin-film bulk through a highly defected material to selectively passivate only $c$-Si surface states. It can be considered therefore, that bulk hydrogen diffusion occurring at higher processing temperatures simply masked the underlying surface passivation mechanism evident in this work.

In conclusion, we used contactless PCD measurements of the effective carrier lifetime following incremental postdeposition thermal annealing to study the surface passivation mechanism for $n$-type $c$-Si. From this we determined that the 
energy for the thermally activated surface passivation was $0.7 \pm 0.1 \mathrm{eV}$, well below the energies reported for the hydrogen diffusion mechanisms. Our results indicate that surface passivation is a reaction-limited surface reconfiguration process instead of the previously accepted bulk hydrogen diffusion process at the $a-\mathrm{Si}: \mathrm{H}(i) / c-\mathrm{Si}(n)$ interface.

The authors wish to thank Murdoch University for use of their PECVD system. This work was supported by the Australian Research Council.

${ }^{1}$ M. Taguchi, K. Kawamoto, S. Tsuge, T. Baba, H. Sakata, M. Morizane, K. Uchihashi, N. Nakamura, S. Kiyama, and O. Oota, Prog. Photovoltaics. 8, 503 (2000)

${ }^{2}$ S. Dauwe, J. Schmidt, and R. Hezel, Proceedings of the 29th IEEE Photovoltaic Specialists Conference, 2002 (unpublished), p. 1246.

${ }^{3}$ B. Lenkeit and R. Hezel, Proceedings of the 17 th European Photovoltaic
Solar energy Conference, 2001 (unpublished), p. 343.

${ }^{4}$ A. Smets, W. Kessels, and M. Van de Sanden, Appl. Phys. Lett. 82, 865 (2003).

${ }^{5}$ R. Street and K. Winer, Phys. Rev. B 40, 6236 (1989).

${ }^{6}$ B. Tuttle, C. Van de Walle, and J. Adams, Phys. Rev. B 59, 5493 (1999).

${ }^{7}$ W. Wampler, S. Myers, and D. Follstaedt, Phys. Rev. B 55, 13319 (1997).

${ }^{8}$ S. Pantelides, Phys. Rev. Lett. 58, 1344 (1987).

${ }^{9}$ D. Schroder, Semiconductor Material and Device Characterization (Wiley, Hoboken, NJ, 2006), p. 399.

${ }^{10}$ S. De Wolf and M. Kondo, Appl. Phys. Lett. 90, 042111 (2007).

${ }^{11}$ D. Biegelson, N. Johnson, M. Stutzmann, E. Poindexter, and P. Caplan, Appl. Surf. Sci. 22, 879 (1985).

${ }^{12}$ B. Sopori, X. Deng, J. Benner, A. Rohatgi, P. Sana, S. Estreicher, Y. Park, and M. Robertson, Sol. Energy Mater. Sol. Cells 41, 159 (1996).

${ }^{13}$ H. Dekkers, S. De Wolf, G. Agostinelli, F. Duerinckx, and G. Beaucarne, Sol. Energy Mater. Sol. Cells 90, 3244 (2006).

${ }^{14}$ J. Dupuis, E. Fourmond, J. Lelièvre, D. Ballutaud, and M. Lemiti, Thin Solid Films 516, 6954 (2008).

${ }^{15}$ A. Aberle, Prog. Photovoltaics 8, 473 (2000). 